\title{
High spatial validity is not sufficient to elicit voluntary shifts of attention
}

\author{
Joseph R. Pauszek ${ }^{1}$ - Bradley S. Gibson ${ }^{1,2}$
}

Published online: 4 April 2016

(C) The Psychonomic Society, Inc. 2016

\begin{abstract}
Previous research suggests that the use of valid symbolic cues is sufficient to elicit voluntary shifts of attention. The present study interpreted this previous research within a broader theoretical context which contends that observers will voluntarily use symbolic cues to orient their attention in space when the temporal costs of using the cues are perceived to be less than the temporal costs of searching without the aid of the cues. In this view, previous research has not addressed the sufficiency of valid symbolic cues, because the temporal cost of using the cues is usually incurred before the target display appears. To address this concern, $70 \%$-valid spatial word cues were presented simultaneously with a search display. In addition, other research suggests that opposing cuedependent and cue-independent spatial biases may operate in these studies and alter standard measures of orienting. After identifying and controlling these opposing spatial biases, the results of two experiments showed that the word cues did not elicit voluntary shifts of attention when the search task was relatively easy but did when the search task was relatively difficult. Moreover, the findings also showed that voluntary use of the word cues changed over the course of the experiment when the task was difficult, presumably because the temporal cost of searching without the cue lessened as the task got easier with practice. Altogether, the present findings
\end{abstract}

Electronic supplementary material The online version of this article (doi:10.3758/s13414-016-1097-4) contains supplementary material, which is available to authorized users.

Bradley S. Gibson

bgibson@nd.edu

1 University of Notre Dame, Notre Dame, IN, USA

2 Department of Psychology, University of Notre Dame, 118 Haggar Hall, Notre Dame, IN 46556, USA suggested that the factors underlying voluntary control are multifaceted and contextual, and that spatial validity alone is not sufficient to elicit voluntary shifts of attention.

Keywords Spatial attention · Spatial cuing, Symbolic attention control $\cdot$ Voluntary attention control

Spatial symbols are often used to elicit voluntary shifts of attention in the laboratory, though these symbols may also elicit involuntary (or automated) shifts of attention (Friesen \& Kingstone, 1998; Friesen, Ristic, \& Kingstone, 2004; Gibson \& Bryant, 2005; Ho \& Spence, 2006; Hommel, Pratt, Colzato, \& Godijn, 2001; Jonides, 1981; Leblanc \& Jolicoeur, 2010; Pratt, Radulescu, Guo, \& Hommel, 2010; Ristic, Friesen, \& Kingstone, 2002; Ristic \& Kingstone, 2006, 2009; Tipples, 2002, 2008). For the past 30 years, the primary tool used to study attentional shifts in response to such symbolic cues has been the spatial cuing paradigm (Posner, 1980; Posner, Snyder, \& Davidson, 1980). In this paradigm, observers are shown visual displays and are required to detect, discriminate, or identify some target stimulus presented in their periphery while keeping their eyes fixated on a central location. To aid in their search for the target, a symbolic cue is often shown at fixation, which correctly identifies the location of the target on some trials (i.e., valid trials) but not on others (i.e., invalid trials). In most variations of the spatial cuing paradigm, there is only one target present in each display, accompanied by visually similar nontarget distractors. Under these conditions, observers can choose to use the cue to locate the target or they can choose to perform an unguided search for the target without the aid of the cue. Spatial symbols are thought to elicit voluntary shifts of attention when observers intentionally use the spatial knowledge that is conveyed by the cue to guide their search. When this occurs, 
significant costs-plus-benefits are typically observed; that is, mean correct response times (RTs) are typically faster on valid trials than on invalid trials.

Within the extensive literature on spatial cuing there has been a long-standing belief that manipulations of the cue's spatial validity can manipulate the voluntary versus involuntary control of attention, with high (or informative) spatial validities eliciting voluntary shifts of attention and low (or uninformative) spatial validities eliciting involuntary shifts of attention (see, e.g., Hommel et al., 2001; see also Folk \& Gibson, 2001; Gibson, Folk, Theeuwes, \& Kingstone, 2008; Wright \& Ward, 2008; Yantis, 1996, for a broader discussion of this issue). However, in the present study, we propose that this perspective grossly oversimplifies the way in which observers voluntarily or not choose to use a spatial cue to orient their attention. While we certainly agree that manipulations of spatial validity can influence observers' decision, we disagree with the notion that voluntary symbolic control should inevitably occur simply because the cue is of a high spatial validity. Rather, we contend that observers' consideration of the spatial validity of the cue feeds into a broader analysis of the task that weighs the perceived temporal cost of using the cue to guide attention versus the perceived temporal cost of searching without the aid of the cue (see also Gray, Sims, Fu, \& Schoelles, 2006, for a similar but more general perspective). In this view, the decision to voluntarily use a symbolic cue would occur not so much because the cue had high spatial validity per se but rather because the temporal cost of comprehending the cue and using it to guide attention was judged to be less than the temporal cost of searching without the aid of the cue (see also Horowitz, Wolfe, Alvarez, Cohen, \& Kuzmova, 2009; Wolfe, Alvarez, \& Horowitz, 2000).

Consistent with this account, Davis and Gibson (2012) recently showed that high spatial validity was not sufficient to elicit the voluntary use of spatial word cues by comparing performance across the spatial cuing tasks depicted in Fig. 1a and $\mathrm{b}$. In both tasks, observers were instructed to use a $100 \%$-valid spatial word cue (above, below, left, or right) presented at fixation to locate and discriminate the identity of the cued target letter ( $H$ or $U$ ). In the ambiguous target context shown in Fig. 1a, all four of the letters shown in the display were target letters; two were the letter $H$ and two were the letter $U$. In this context, use of the cue was considered to be mandatory because it was the only basis for disambiguating the one true target letter from the three false target letters. In the unambiguous target context shown in Fig. 1b, only one target letter was shown in each display along with three visually similar nontarget letters. In this context, use of the cue was considered to be voluntary because observers could either choose to use the $100 \%$-valid cue to shift their attention directly to the target or they could choose to ignore the cue and freely search for the only target letter present in each display.
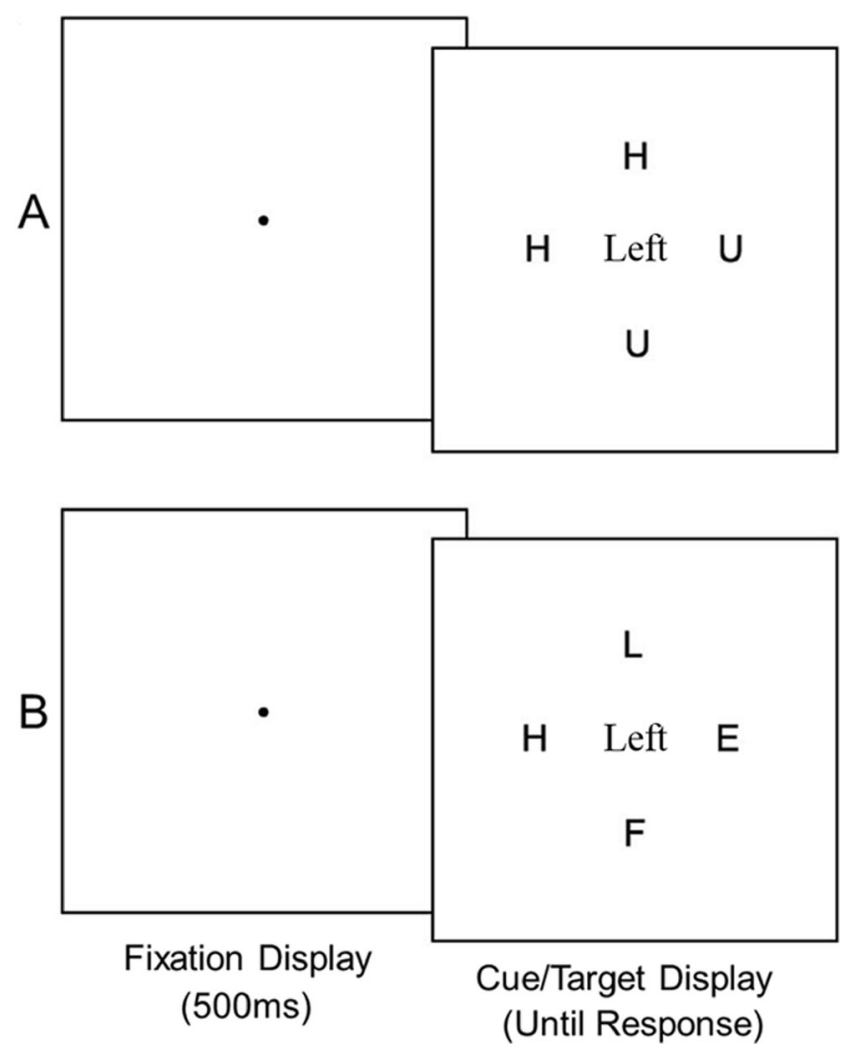

Fig. 1 Typical display sequences used by Davis and Gibson (2012). Panel A depicts the ambiguous (mandatory) target displays; Panel B depicts the unambiguous (voluntary) target displays. The spatial word cue was $100 \%$ valid for both types of target displays, and the task was to discriminate the identity $(H$ or $U$ ) of the target. The figure is not drawn to scale

Because a $100 \%$-valid cue does not warrant the use of costs-plus-benefits to measure shifts of attention, Davis and Gibson (2012) relied instead on the "cued axis effect" as an empirical marker of voluntary symbolic control. The cued axis effect has regularly been used as a direct measure of cue processing, and it has become diagnostic of the comprehension of these spatial terms across a variety of experimental paradigms (Bryant, Tversky, \& Franklin, 1992; Carlson \& Van Deman, 2008; Corballis, 1988; Franklin \& Tversky, 1990; Logan, 1995; Logan \& Sadler, 1996). Specifically, spatial words such as above and below can be comprehended faster than spatial words such as left or right because the vertical axis of space can be accessed prior to the horizontal axis of space during the process of cue comprehension (Logan, 1995). In contrast, the comprehension of other spatial symbols such as arrows and eye gaze do not appear to be marked by the cued axis effect, presumably because they require a less effortful and more basic form of processing (Gibson \& Kingstone, 2006; Gibson, Scheutz, \& Davis, 2009). Based on these findings, Davis and Gibson (2012) focused exclusively on spatial word cues and used the presence versus absence of the cued axis effect to measure the presence versus absence of voluntary symbolic control, respectively. 
When the ambiguous and unambiguous target displays were presented in separate trial blocks and shown simultaneously with the $100 \%$-valid spatial word cues, Davis and Gibson (2012) observed the cued axis effect when the target context was ambiguous and use of the cue was mandatory (as in Fig. 1a), but they failed to observe this effect when the target context was unambiguous and use of the cue was voluntary (as in Fig. 1b). In addition, Davis and Gibson showed that the cued axis effect could be observed in the unambiguous display condition when these displays were randomly intermixed with the ambiguous displays, thus forcing observers to adopt a mandatory cue processing strategy across both display conditions. These findings suggested that the presence versus absence of the cued axis effect in the unambiguous condition varied as a function of the extent to which cue processing was mandatory versus voluntary, respectively, and suggested that when cue processing was voluntary, observers preferred to search for the target without the aid of the spatial word cues.

Moreover, Davis and Gibson (2012) showed that the cued axis effect could also be observed when the unambiguous displays were blocked so long as a temporal delay was inserted between presentation of the spatial word cue and the target display. Although this cue-target stimulus onset asynchrony (SOA) has been inserted in the vast majority of spatial cuing experiments, Davis and Gibson suggested that it changed the temporal dynamics of the decision to search with or without the aid of the cue in a way that might incentivize cue processing. Instead of one decision to search with or without the aid of the cue, there were now two decisions facing the observer. Upon appearance of the cue, the first decision was to process the cue or do nothing task related (because the target display had not yet appeared); and, upon appearance of the target display, the second decision was to apply the spatial knowledge gained from the cue to search for the target (assuming it had been processed) or to search without the aid of this knowledge. In other words, insertion of this cue-target SOA might incentivize cue processing because the temporal cost of processing the cue could be incurred before the appearance of the target display. Hence, they concluded that voluntary shifts of attention are best evaluated when the cue and target display are presented simultaneously (i.e., when the cue-target SOA is $0 \mathrm{~ms}$ ).

The findings reported by Davis and Gibson (2012) have important implications for theories of attention control. However, their interpretations were largely grounded in the assumption that a nonsignificant cued axis effect reflected a lack of cue comprehension. It is possible, though, that the operation of other spatial biases could have complicated their interpretations. For instance, in their series of experiments using $25 \%$-valid (i.e., uninformative) head and arrow cues and a simple detection task, Nomura, Katahata, and Hashiya (2005) concluded that spatial orienting occurs asymmetrically along the horizontal and vertical planes, with a bias to shift attention along the horizontal axis of space. As a possible explanation for their findings, the authors referred to Kobayashi and Kohshima (1997; 2001a, 2001b), who suggested that a bias facilitating attentional orienting along the horizontal axis occurs ubiquitously due to the evolutionary development of the eye outline in humans, which is elongated in the horizontal plane and presumably reflects humans' niche as a terrestrial species (see also Carrasco, Talgar, \& Cameron, 2001; Corbett \& Carrasco, 2011; Robertson, 2004). If this spatial bias is based on the anatomical structure of the eye, then it likely operates independent of the meaning of the cue and may be most apparent on trials in which the cue is not fully processed. Hence, there may be two directly opposing axis effects that operate in the spatial cuing paradigm; when spatial word cues are shown, the above/below advantage associated with the cued axis effect reflects cue-dependent processing and is likely most apparent when observers use the spatial word cues to orient their attention, while the left/right advantage reflects cue-independent processing and is likely most apparent when observers are freely searching the displays.

The purpose of the present study was twofold. First and foremost, the present study was aimed at strengthening and expanding a novel hypothesis of voluntary symbolic control. According to this hypothesis, observers weigh the perceived temporal costs of using symbolic cues to guide their attention versus the perceived temporal costs of searching without the aid of the cue. This account suggests that, contrary to the longstanding assumption, observers may choose to ignore a spatial symbol with high spatial validity, provided that the temporal costs of freely searching the display are perceived to be less than the temporal costs of using the cue to guide their attention. Novel evidence for this account will be provided in Experiment 3.

However, a hypothesis is only as good as the empirical data that supports it. Although Davis and Gibson (2012) recently found support for this view, it is possible that there may have been an underlying spatial bias, the left/right advantage, operating in their study which could have complicated their interpretations. For this reason, the second aim of the present study was to seek further evidence for the left/right advantage associated with cue-independent processing and to understand the extent to which this spatial bias may alter commonly used measures of cue processing. If an underlying left/right advantage can affect the cued axis effect and costs-plus-benefits, then the way in which spatial cuing experiments are typically designed and analyzed may need to be reconsidered. Accordingly, the second aim of the present study was to devise a measure of voluntary control that was unbiased by the left/right advantage. These issues were addressed in Experiments 1 and 2.

\section{Experiment 1}

Experiment 1 was conducted to examine the extent to which observers prefer to search for targets on the horizontal axis 
versus the vertical axis when no cue is made available. If a cue-independent horizontal bias can occur under these conditions, then a significant left/right advantage should be observed in the present experiment.

\section{Method}

Participants Twenty University of Notre Dame undergraduates participated to partially fulfill a course requirement. All participants reported normal or corrected-tonormal acuity and normal color vision.

Stimuli and apparatus A personal computer running DMDX software (Forster \& Forster, 2003) with a 17-inch CRT monitor was used for stimulus presentation and data collection. Responses were collected using a custom-built response box with response time rounded to the nearest millisecond. A fixed viewing distance of $57 \mathrm{~cm}$ was enforced using a chin rest, and the experiment was conducted in a dimly lit room. Each trial consisted of two displays presented on a black background: a fixation display and a target display. The fixation display consisted of a small white dot in the center of the screen $\left(0.38^{\circ}\right.$ in diameter). The target display contained four letters, presented in red, $4.37^{\circ}$ from fixation in each of the four cardinal directions. Each letter was $1.51^{\circ}$ tall and $0.75^{\circ}$ wide. Of the four letters that were shown on each trial, one was always the target $(H$ or $U$ ); the remaining three letters were non-targets that were randomly chosen without replacement from among the following seven letters: $S, P, L, C, E, A, F$.

Procedure The display sequence was similar to that shown in Fig. $1 \mathrm{~b}$ with the sole exception being that a spatial word cue was not shown. Instead, each trial began with a fixation display for $500 \mathrm{~ms}$ followed immediately by the appearance of the target display. Both the fixation dot and target display remained on the screen until a response was made (or until 4,000 ms elapsed). The target appeared at each of the four potential target locations equally often and on each trial, the target location was equally likely to contain either an $H$ or $U$. There were 16 practice trials followed by a total of 704 experimental trials arranged into two blocks. Participants were given the opportunity to take a total of four self-timed breaks across the duration of the experiment; each opportunity occurred after 176 trials. The participants' task was to locate and discriminate the identity of the target letter as quickly and accurately as possible. Participants always used their left hand to respond " $\mathrm{H}$ " and their right hand to respond " $\mathrm{U}$ ".

\section{Results and discussion}

Mean correct RTs and percentage error rates are listed in Table 1 as function of target location. Note that in this, and all subsequent experiments, mean correct RTs greater than or
Table 1 Mean correct response times (RTs) (ms) and error rates (\%) for Experiment 1 listed as a function of target location. Standard errors are shown in parentheses

\begin{tabular}{lll}
\hline & RT $(S E)$ & Errors $(S E)$ \\
\hline Target location & & \\
Above & $624(33.23)$ & $3.67(0.67)$ \\
Below & $641(29.95)$ & $3.70(0.54)$ \\
Left & $590(30.95)$ & $3.74(0.58)$ \\
Right & $578(26.87)$ & $3.06(0.41)$ \\
\hline
\end{tabular}

equal to 3.0 standard deviations from the overall mean of each participant were trimmed to that value. Approximately $1.70 \%$ of the RTs in Experiment 1 were trimmed in this fashion.

To investigate whether a cue-independent left/right advantage can occur in this search task, a paired-samples $t$ test was conducted comparing mean correct RTs in response to targets on the vertical and horizontal axes. The results indicated that RTs were significantly faster when the target appeared on the horizontal axis $(M=584 \mathrm{~ms})$ than when the target appeared on the vertical axis $(M=632 \mathrm{~ms}), t(19)=7.96, p<.001, d=.36$. An identical analysis conducted on percentage error rates indicated no significant difference $(M=3.40$ and $M=3.68$, respectively), $t(19)=.86, p=.400, d=.12$. These findings suggest that a cue-independent spatial bias operates in this search task and facilitates shifts of attention along the horizontal axis. We also replicated this finding in each of two additional studies involving independent samples of 20 observers each (the studies were otherwise identical to Experiment 1).

Thus far, we have construed the left/right advantage as arising from the physical structure of the eye (Kobayashi \& Kohshima, 1997, 2001a, b). In this view, there is no reason to expect that shifts of attention along the horizontal axis would be biased in one direction or the other. However, a recent attentional model introduced by Takio, Koivisto, and Hämäläinen (2014) suggests that a right visual field advantage occurs in both linguistic and nonlinguistic spatial attention tasks due to lateralized processing of linguistic stimuli, as well as asymmetries in attentional, bottom-up neural processes (see also Robertson, 2004). This account can be further distinguished from other higher level, cultural accounts, which suggest a left-to-right attentional bias in a variety of different paradigms, though the interpretations of this bias have been somewhat mixed (see Ossandon, Onat, \& Konig, 2014, for a review). One interpretation suggests that visuospatial asymmetries along the horizontal axis are critically modulated by directional oculomotor routines associated with reading and writing. Specifically, it has been suggested that observers who read from left to right have two established tendencies when presented with a line of print or linguistic stimuli in both visual fields (Heron, 1957; see also, Rinaldi, Di Luca, Henik, \& Girelli, 2014; Spalek \& Hammad, 2005). The first tendency 
is to fixate or establish attention near the beginning of the line of text, which is typically to the left. The second tendency is to shift attention along the line of print from left to right. Thus, if observers begin their unguided search by orienting attention toward the typical starting point of text, then the left/right advantage could be driven by a leftward attentional bias. On the other hand, if observers begin their unguided search by shifting attention rightward, away from the starting point of central fixation, then the left/right advantage could be driven by a rightward attentional bias. Dickson and Intraub (2009) reported a similar left-to-right attentional bias when observers viewed to-be-remembered objects in complex visual scenes.

To investigate whether the left/right advantage found in the present study is primarily driven by a leftward or rightward attentional bias, a subsequent paired-samples $t$ test was conducted comparing mean correct RTs in response to targets at the left versus right locations. No significant difference was found between RTs to targets at the left $(M=590 \mathrm{~ms})$ and right $(M=578 \mathrm{~ms})$ locations, $t(19)=1.71, p=.104, d=.09$. Moreover, RTs in the target left condition were significantly faster than RTs in both the target above condition $(M=624$ $\mathrm{ms}), t(19)=4.99, p<.001, d=.24$, and the target below condition $(M=641 \mathrm{~ms}), t(19)=6.95, p<.001, d=.37$. Likewise, RTs in the target right condition were significantly faster than RTs in both the target above condition, $t(19)=4.80$, $p<.001, d=.34$, and the target below condition, $t(19)=7.42$, $p<.001, d=.49$. Identical analyses conducted on percentage error rates revealed no significant differences between any two target locations (all $p \mathrm{~s}>.080$ ). These findings suggest that the left/right advantage is not systematically driven by faster RTs or fewer errors to targets appearing at the left or right locations alone.

In summary, the results obtained in Experiment 1 suggest that a cue-independent spatial bias speeds attentional orienting along the horizontal axis relative to the vertical axis.

\section{Experiment 2}

The cue-independent left/right advantage observed in Experiment 1 could have offset the magnitude of the cued axis effect observed in Davis and Gibson's (2012) study. If this true, then their conclusion that high spatial validity was not sufficient to elicit voluntary symbolic control needs to be reevaluated. One way to resolve this issue is to provide a second measure of cue comprehension in addition to the cued axis effect. Indeed, most previous studies have inferred the elicitation of voluntary shifts of attention by measuring the costs-plus-benefits of orienting attention in accordance with the cue. Davis and Gibson (2012) could not measure costsplus-benefits because the spatial word cues used in their study were always valid. Accordingly, the validity of the spatial word cues was reduced from $100 \%$ to $70 \%$ in Experiment 2 .
This value was chosen because it was highly valid while also providing an adequate number of trials in the invalid condition. Previous research has suggested that valid spatial word cues such as these can elicit significant costs-plus-benefits (Mayer \& Kosson, 2004; Vecera \& Rizzo, 2004); however, these previous studies used only non-zero cue-target SOAs, which may have provided an incentive to process the cues (Davis \& Gibson, 2012). Thus, it is important to examine whether these highly valid spatial word cues can elicit voluntary shifts of attention when this cue-processing incentive is minimized, as when the cue-target SOA is $0 \mathrm{~ms}$.

One important advantage to using costs-plus-benefits to measure the voluntary control of attention is that the influence of the cue-independent left/right advantage could be neutralized by including only those valid and invalid trials that came from the same axis (i.e., horizontal invalid-horizontal valid or vertical invalid-vertical valid). These unbiased costs-plusbenefits should produce evidence for voluntary shifts of attention only when observers used the spatial word cues to guide their attention. In contrast, biased costs-plus-benefits included valid and invalid trials from different axes. Exclusion of these costs-plus-benefits was important because they could produce artefactual evidence for voluntary shifts of attention even though no cue processing had occurred. Unlike the unbiased costs-plus-benefits, the direction of the biased costs-plusbenefits depended on the extent to which the valid and invalid trials were compatible or incompatible with the left/right advantage. For instance, the direction of biased compatible costs-plus-benefits should be positive, even in the absence of cue processing, when the validly cued target appeared on the horizontal axis and the invalidly cued target appeared on the vertical axis (i.e., horizontal valid RTs should be faster than vertical invalid RTs due to the left/right advantage); whereas, the direction of biased incompatible costs-plus-benefits should be negative when the validly cued target appeared on the vertical axis and the invalidly cued target appeared on the horizontal axis (i.e., vertical valid RTs should be slower than horizontal invalid RTs due to the left/right advantage). Finally, the overall costs-plus-benefits, reflecting an average of all three types, will also be reported to examine how the failure to acknowledge the left/right advantage can lead to misleading conclusions about the occurrence of voluntary symbolic control. A summary of these different types of costs-plus-benefits is provided in Table 2.

\section{Method}

Participants Twenty University of Notre Dame undergraduates participated to partially fulfill a course requirement. All participants reported normal or corrected-to-normal acuity and normal color vision. 
Table 2 An outline of the categories of costs-plus-benefits reported in Experiments 2 and 3 of the present study

\begin{tabular}{|c|c|c|c|c|c|}
\hline \multirow{2}{*}{$\begin{array}{l}\text { Category of } \\
\text { costs-plus-benefits }\end{array}$} & \multirow[t]{2}{*}{ Cued direction } & \multirow[t]{2}{*}{ Uncued direction } & \multicolumn{2}{|c|}{ Compatibility with the left/right advantage } & \multirow{2}{*}{$\begin{array}{l}\text { Measurement of costs-plus-benefits } \\
\text { due to the left/right advantage }\end{array}$} \\
\hline & & & Cued Direction & Uncued Direction & \\
\hline Biased compatible & Left/right & $\begin{array}{l}\text { Orthogonal } \\
\text { (Target on vertical axis) }\end{array}$ & Compatible & Incompatible & Overestimated \\
\hline Biased incompatible & Above/below & $\begin{array}{l}\text { Orthogonal } \\
\text { (Target on horizontal axis) }\end{array}$ & Incompatible & Compatible & Underestimated \\
\hline Unbiased A/B & Above/below & $\begin{array}{l}\text { Opposite } \\
\text { (Target on vertical axis) }\end{array}$ & Incompatible & Incompatible & Unbiased \\
\hline Unbiased L/R & Left/right & $\begin{array}{l}\text { Opposite } \\
\text { (Target on horizontal axis) }\end{array}$ & Compatible & Compatible & Unbiased \\
\hline
\end{tabular}

Stimuli and apparatus The stimuli and apparatus used in Experiment 2 were identical to those used in Experiment 1 with one exception: namely, a central spatial word cue, presented in white, was also included at fixation in the target displays. The four spatial word cues were Above, Below, Left and Right. Each spatial word cue was $0.68^{\circ}$ tall and varied in width from $1.18^{\circ}$ to $1.94^{\circ}$.

Procedure A typical display sequence is shown in Fig. $1 \mathrm{~b}$. Each trial began with a fixation display for $500 \mathrm{~ms}$ followed immediately by simultaneous appearance of the spatial word cue and the target display. The cues indicated the correct target location on approximately $70 \%$ of trials. The spatial word cue referred to each of the four potential target directions equally often. On valid trials, the cued direction was equally likely to contain an $H$ or $U$. On invalid trials, the target display was also equally likely to contain an $H$ or $U$, and the target was equally likely to appear at one of the three remaining locations; one of these locations was opposite to the cued direction and the other two locations were orthogonal to the cue direction. The target display remained on the screen until a response was made (or until 4,000 ms elapsed).

There were 16 practice trials followed by a total of 704 experimental trials arranged into two blocks. Within each block, there were 64 validly cued trials for each of the four spatial cues for a total of $64 \times 4=256$ valid trials, and 24 associated invalidly cued trials ( 8 opposite the cued direction and 16 orthogonal to the cued direction) for a total of $24 \times 4=$ 96 invalid trials. The order of trials within each block was randomized, and a different random order was presented to each participant. Participants were informed that the spatial word cue would indicate the correct direction of the target on approximately $70 \%$ of the trials. Participants were given the opportunity to take a total of four self-timed breaks across the duration of the experiment; each opportunity occurred after 176 trials. The participants' task was to discriminate the identity of the target letter ( $H$ or $U$ ) as quickly and accurately as possible. Participants always used their left hand to respond "H" and their right hand to respond "U." Note that Gibson and Kingstone (2005) investigated potential interactions between response orientation (response keys on the right or left of the response pad vs. on the top or bottom of the response pad) and cue-dependent axis but found no evidence of either facilitation or interference when, for example, observers had to orient in the left cued direction and respond using the left or right key, respectively, relative to using the top or bottom key (see also Gibson \& Davis, 2011).

\section{Results and discussion}

Mean correct RTs and percentage error rates are shown in Table 3 as a function of cue validity and cued axis. Approximately $1.60 \%$ of the RTs were trimmed according to the procedure described in Experiment 1. Point estimates for the overall, unbiased, and biased costs-plus-benefits are shown in Fig. 2 along with 95\% confidence intervals (CIs), based on a within-subjects design.

Axis effects The above/below advantage associated with the cued axis effect and the opposing left/right advantage were examined by focusing exclusively on validly cued trials. A paired-samples $t$ test was conducted on mean correct RTs and percentage error rates to examine the difference between the above/below and left/right conditions. With respect to

Table 3 Mean correct response times (RTs) (ms) and error rates (\%) for Experiment 2 listed as a function of cue-dependent axis and cue validity. Standard errors are shown in parentheses

\begin{tabular}{lll}
\hline & RT $(S E)$ & Errors $(S E)$ \\
\hline $\begin{array}{l}\text { Cue-dependent axis } \\
\text { Above/below }\end{array}$ & \\
$\quad$ Cued & $638(31.91)$ & $3.88(0.59)$ \\
$\quad$ Uncued opposite & $648(31.80)$ & $4.55(0.77)$ \\
$\quad$ Uncued orthogonal & $599(33.64)$ & $3.34(0.62)$ \\
Left/right & & \\
$\quad$ Cued & $595(32.68)$ & $4.17(0.56)$ \\
$\quad$ Uncued opposite & $624(46.53)$ & $4.58(1.24)$ \\
$\quad$ Uncued orthogonal & $668(44.61)$ & $4.76(0.66)$ \\
\hline
\end{tabular}






Fig. 2 Costs-plus-benefits (invalid RT-valid RT) in the overall, biased compatible, biased incompatible, unbiased above/below, and unbiased left/right categories of Experiment 2. Error bars represent the 95\% confidence intervals based on a within-subjects design

mean correct RTs, the results indicated that RTs were significantly faster in the left/right condition $(M=595 \mathrm{~ms})$ than in the above/below condition $(M=638 \mathrm{~ms}), t(19)=5.72, p<$ $.001, d=.30$. An identical analysis conducted on percent error rates indicated no significant difference $(M=4.17$ and $M=$ 3.88 , respectively), $t(19)=.82, p=.425, d=.11$. The presence of this significant left/right advantage is consistent with the results of Experiment 1 and suggests that cue-independent spatial biases dominated performance in this experiment. If this interpretation is correct, then costs-plus-benefits should be nonsignificant when the influence of this cue-independent spatial bias is neutralized in the unbiased condition.

Overall costs-plus-benefits A paired-samples $t$ test was conducted on mean correct RTs and percentage error rates to examine the difference between all valid and all invalid trials. With respect to mean correct RTs, valid RTs $(M=617 \mathrm{~ms})$ were significantly faster than invalid RTs ( $M=634 \mathrm{~ms}), t(19)$ $=2.39, p=.028, d=.11$. An identical analysis conducted on percentage error rates indicated no significant difference between the valid and invalid conditions $(M=4.04$ and $M=$ 4.21 , respectively), $t(19)=.46, p=.654, d=.07$. At first glance, these findings suggest that observers were processing the cues and using them to orient their attention. However, these overall costs-plus-benefits do not account for the potential influence of the cue-independent left/right advantage.

Biased compatible costs-plus-benefits Paired-samples $t$ tests were conducted on mean correct RTs and percentage error rates to examine the differences between valid and invalid trials when the valid trials came from the horizontal axis and the associated invalid trials came from the vertical axis. With respect to mean correct RTs, valid RTs $(M=595 \mathrm{~ms})$ were significantly faster than invalid RTs $(M=668 \mathrm{~ms}), t(19)=$ $4.99, p<.001, d=.42$. An identical analysis conducted on percentage error rates indicated no significant difference between the valid and invalid conditions $(M=4.17$ and $M=$ 4.76, respectively), $t(19)=.94, p=.359, d=.22$. Although significant positive costs-plus-benefits were observed in this condition, the source of these effects was ambiguous and could have arisen from the left/right advantage, the meaning of the cues, or a combination of both.

Biased incompatible costs-plus-benefits Paired-samples $t$ tests were conducted on mean correct RTs and percentage error rates to examine the differences between valid and invalid trials when the valid trials came from the vertical axis and the associated invalid trials came from the horizontal axis. With respect to mean correct RTs, valid RTs $(M=638)$ were significantly slower than invalid RTs $(M=599 \mathrm{~ms}), t(19)=$ $4.49, p<.001, d=.27$. An identical analysis conducted on percentage error rates indicated no significant difference between the valid and invalid conditions $(M=3.88$ and $M=$ 3.35 , respectively), $t(19)=1.09, p=.290, d=.20$. The significant negative costs-plus-benefits observed in this condition suggest that the orientation of attention was influenced more by the left/right advantage than by the meaning of the cues.

Unbiased costs-plus-benefits Paired-samples $t$ tests were conducted on mean correct RTs and percentage error rates to examine the differences between valid and invalid trials from the same axis. With respect to mean correct RTs, the results indicated that the difference between the valid and invalid conditions was nonsignificant along the vertical axis $(M=$ $638 \mathrm{~ms}$ and $M=648 \mathrm{~ms}$, respectively), $t(19)=1.11, p=$ $.279, d=.07$, and along the horizontal axis $(M=595 \mathrm{~ms}$ and $M=624 \mathrm{~ms}$, respectively), $t(19)=1.85, p=.081, d=$ .16. Identical analyses conducted on percentage error rates also indicated that the difference between the valid and invalid conditions was nonsignificant along the vertical axis $(M=$ 3.88 and $M=4.55$, respectively), $t(19)=1.05, p=.309, d=$ .22 , and along the horizontal axis $(M=4.17$ and $M=4.58$, respectively), $t(19)=.33, p=.746, d=.09$. These findings suggest that the orientation of attention was not significantly influenced by the meaning of the cues when the cueindependent left/right advantage was neutralized.

Summary The results of Experiment 2 provided evidence that the left/right advantage can dominate performance in the spatial cuing paradigm when the spatial validity of the spatial word cues was approximately $70 \%$. This finding is important because it reflects a relative lack of cue processing and is opposite to the above/below advantage associated with the cued axis effect that is expected when a spatial word cue is processed. Perhaps more importantly, the results of Experiment 2 also demonstrated the importance of evaluating costs-plus-benefits within the same spatial axis so as to neutralize the cue-independent left/right advantage. When the cue-independent left/right advantage was properly controlled using unbiased costs-plus-benefits, the main findings suggested that the $70 \%$-valid spatial word cues had little to no 
influence over the orientation of attention. In our view, the spatial word cues had little to no influence over the orientation of attention because the temporal cost of searching without the cue was judged to be less than the temporal cost of searching with the cue.

\section{Experiment 3}

The empirical findings reported in Experiments 1 and 2 have important implications for understanding how to best measure voluntary shifts of attention in the spatial cuing paradigm when more than a single axis is utilized. Experiment 3 used this knowledge to advance understanding of voluntary symbolic control. Accordingly, Experiment 3 focused exclusively on unbiased costs-plus-benefits (see supplementary materials for a detailed analysis of axis effects and overall, biased compatible, and biased incompatible costs-plus-benefits). In our view, the decision to voluntarily use a symbolic cue occurs when the temporal cost of comprehending the cue and using it to guide attention is judged to be less than the temporal cost of searching without the aid of the cue. Observers' willingness to use the cues was increased in Experiment 3 not by increasing the spatial validity of cue but rather by increasing the temporal costs of searching without the cue. Specifically, as observers weigh the temporal costs of using symbolic cues to guide attention versus the temporal costs of searching without the aid of the cue, it is reasonable to hypothesize that they will be more willing to process and use the $70 \%$-valid spatial word cues to orient their attention when the search task is made more difficult. Thus, Experiment 3 manipulated the difficulty of the search task (easy vs. hard).

In the easy task context, we expected to replicate the findings obtained in Experiment 2; namely, we expected to observe a relative lack of unbiased costs-plus-benefits. In contrast, in the hard task context, we expected that observers should be more likely to voluntarily use the $70 \%$-valid spatial word cues to guide their attention because performing an unguided search in this context was more difficult. As such, the magnitude of the unbiased costs-plus-benefits should increase in this context. In addition, because the relative difficulty of these two search tasks might change with practice, we also examined how the magnitude of costs-plus-benefits might change across the duration of the experiment. Accordingly, the experimental trials were divided into three equal blocks, and this within-subjects factor was included in the analyses.

\section{Method}

Participants Forty University of Notre Dame undergraduates participated to partially fulfill a course requirement. Twenty participants were randomly assigned to each of the two task context conditions. All participants reported normal or corrected-to-normal acuity and normal color vision.

Stimuli and apparatus The apparatus was identical to the previous two experiments. The stimuli used in the easy task context condition were similar to those used in Experiment 2 with the following exceptions: All four of the letters in the target display appeared red for half of the participants and they all appeared green for the other half. In addition, the two target letters were $E$ and $U$ and the nontarget letters were always $S, P$, and $A$. In the hard task context condition, the two target letters were $E$ and $U$, as well; however, these target letters were also defined with respect to their orientation (upright or rotated $90^{\circ}$ counterclockwise) and color (red or green). Specifically, the target letter was defined as a red, upright $E$ or $U$ for half of the participants, and as a green, upright $E$ or $U$ for the other half. This task context was more difficult because each of the three nontarget letters differed from a potential target letter in only one of these three specified dimensions. Of the nontarget letters, one was always rotated $90^{\circ}$ counterclockwise, one was always the opposite color of the target letter, and one was always an $S$. For instance, consider the display shown in Fig. 3. In this case, the target is a red, upright $E$. Notice that three of the letters are red, three of the letters are upright, and three of the letters have the identity of one of the target letters. However, only one letter - the target—satisfies all three of these dimensions.

Procedure The procedure was identical to Experiment 2. In both task contexts, there were 10 practice trials followed by a total of 720 experimental trials arranged into three blocks. Within each block, there were 42 validly cued trials for each of the 4 spatial cues for a total of 168 valid trials, and 18 associated invalidly cued trials (6 opposite the cued direction

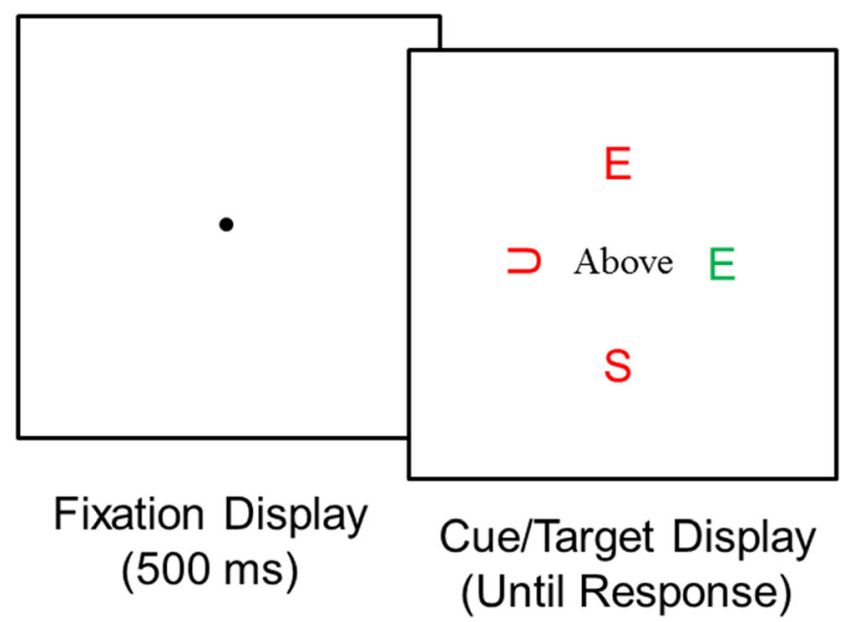

Fig. 3 Typical display sequence used in the hard task context of Experiment 3. The target was defined along three dimensions: identity, color, and orientation. The spatial word cue was $70 \%$ valid, and in this figure the target was the upright, red letter $E$. The figure is not drawn to scale. (Color figure online.) 
and 12 orthogonal to the cued direction) for a total of 72 invalid trials. The order of trials was randomized and a different random order was presented to each participant. Participants were informed that the spatial word cue would indicate the correct direction of the target on $70 \%$ of trials. Participants were given the opportunity to take a self-timed break after each block of trials. Participants always used their left hand to respond "E" and their right hand to respond "U."

\section{Results and discussion}

Mean correct RTs and percentage error rates are shown in Table 4 as a function of cue validity, cued axis, and block in each of the two task context conditions. Approximately 1.50\% of the RTs were trimmed from the easy task context and 1.70\% from the hard task context according to the procedure described in Experiment 1. Point estimates for the overall, unbiased, and biased costs-plus-benefits are shown in Fig. 4 as a function of block along with $95 \%$ confidence intervals (CIs), based on a within-subjects design, in each of the two task contexts. In addition, point estimates for the difference between the easy and hard task contexts in each of these categories are depicted in Fig. 5, along with their associated $95 \%$ confidence intervals, based on a between-subjects design, in each of the three blocks. The error rates in this experiment were generally low and mirrored the findings of Experiments 1 and 2. We therefore focused all of the following analyses and discussion on the RT results.
Unbiased costs-plus-benefits To examine unbiased costsplus-benefits, the difference between mean correct RTs on valid and invalid trials from the same axis were compared within each block of each task context. A four-way, mixed ANOVA was conducted with cued axis (vertical vs. horizontal), validity (valid vs. invalid), and block (Block 1 vs. Block 2 vs. Block 3) as the three within-subjects factors and with task context (easy vs. hard) as the sole betweensubjects factor. The results of this analysis are displayed in Table 5. The significant main effect of cued axis indicated the expected left/right advantage, but cued axis was not included in any significant interactions. Critically, all other lower-order main effects and two-way interactions were qualified by the highest-order significant three-way interaction between validity, block, and task context, $F(2,76)$ $=4.11, p=.020, \eta_{\mathrm{p}}^{2}=.10$. The nature of this three-way interaction was examined further by analyzing the relation between validity and block in each of the two task contexts separately.

In the easy task context, there was a significant main effect of block, $F(2,38)=11.14, p<.001, \eta_{\mathrm{p}}{ }^{2}=.37$, indicating that RTs decreased across the duration of the experiment. However, neither the main effect of validity, $F(1,19)=3.37$, $p=.082, \eta_{\mathrm{p}}^{2}=.15$, nor the interaction between validity and block, $F(2,38)=2.21, p=.123, \eta_{\mathrm{p}}^{2}=.10$, were significant. This finding suggests that little to no cue comprehension occurred in any block of the easy task context.

Table 4 Mean correct response times (RTs) (ms) and error rates (\%) for Experiment 3 listed as a function of cue-dependent axis, cue validity, and block in each of the easy task context and hard task context conditions. Standard errors are shown in parentheses

\begin{tabular}{|c|c|c|c|c|c|c|}
\hline & \multicolumn{2}{|l|}{ Block 1} & \multicolumn{2}{|l|}{ Block 2} & \multicolumn{2}{|l|}{ Block 3} \\
\hline & $\mathrm{RT}(S E)$ & Errors $(S E)$ & $\mathrm{RT}(S E)$ & Errors $(S E)$ & $\mathrm{RT}(S E)$ & Errors $(S E)$ \\
\hline Cue-dependent axis & \multicolumn{6}{|c|}{ Easy task context } \\
\hline \multicolumn{7}{|l|}{ Above/below } \\
\hline Cued & $642(29.72)$ & $3.76(1.23)$ & $598(24.23)$ & $4.98(1.40)$ & $586(20.36)$ & $4.16(1.28)$ \\
\hline Uncued opposite & $668(39.36)$ & $5.42(2.29)$ & $634(27.30)$ & $4.18(0.96)$ & $595(25.71)$ & $3.55(1.06)$ \\
\hline Uncued orthogonal & $617(32.44)$ & $4.80(2.25)$ & $552(24.73)$ & $4.42(0.77)$ & 547 (19.99) & $5.90(1.20)$ \\
\hline \multicolumn{7}{|l|}{ Left/right } \\
\hline Cued & $594(26.23)$ & $5.90(1.20)$ & $563(22.76)$ & $3.88(0.74)$ & $549(21.23)$ & $4.54(0.68)$ \\
\hline Uncued opposite & $624(39.00)$ & $3.74(1.28)$ & $550(25.53)$ & $2.49(0.87)$ & $546(23.87)$ & $4.16(1.30)$ \\
\hline \multirow[t]{2}{*}{ Uncued orthogonal } & $680(40.74)$ & $4.38(1.56)$ & $620(29.60)$ & $3.75(1.00)$ & $596(26.95)$ & $6.68(1.10)$ \\
\hline & \multicolumn{6}{|c|}{ Hard task context } \\
\hline \multicolumn{7}{|l|}{ Above/below } \\
\hline Cued & $1010(48.16)$ & $3.76(0.63)$ & $899(45.86)$ & $3.94(0.84)$ & $864(46.13)$ & $5.00(0.91)$ \\
\hline Uncued opposite & $1233(82.95)$ & $2.50(1.06)$ & $1007(62.71)$ & $2.91(1.09)$ & $910(59.52)$ & $5.40(1.51)$ \\
\hline Uncued orthogonal & $1173(75.98)$ & $3.55(0.97)$ & $924(58.79)$ & $2.30(0.93)$ & 854 (59.99) & $3.96(1.26)$ \\
\hline \multicolumn{7}{|l|}{ Left/right } \\
\hline Cued & $999(51.03)$ & $4.96(0.91)$ & $839(41.66)$ & $4.30(0.69)$ & $793(46.06)$ & $5.42(0.84)$ \\
\hline Uncued opposite & $1108(72.70)$ & $5.00(1.41)$ & $945(71.57)$ & $4.57(1.41)$ & $818(52.67)$ & $5.00(1.64)$ \\
\hline Uncued orthogonal & $1246(79.86)$ & $3.13(0.73)$ & $986(58.24)$ & $6.04(1.02)$ & 937 (66.16) & $7.71(1.66)$ \\
\hline
\end{tabular}




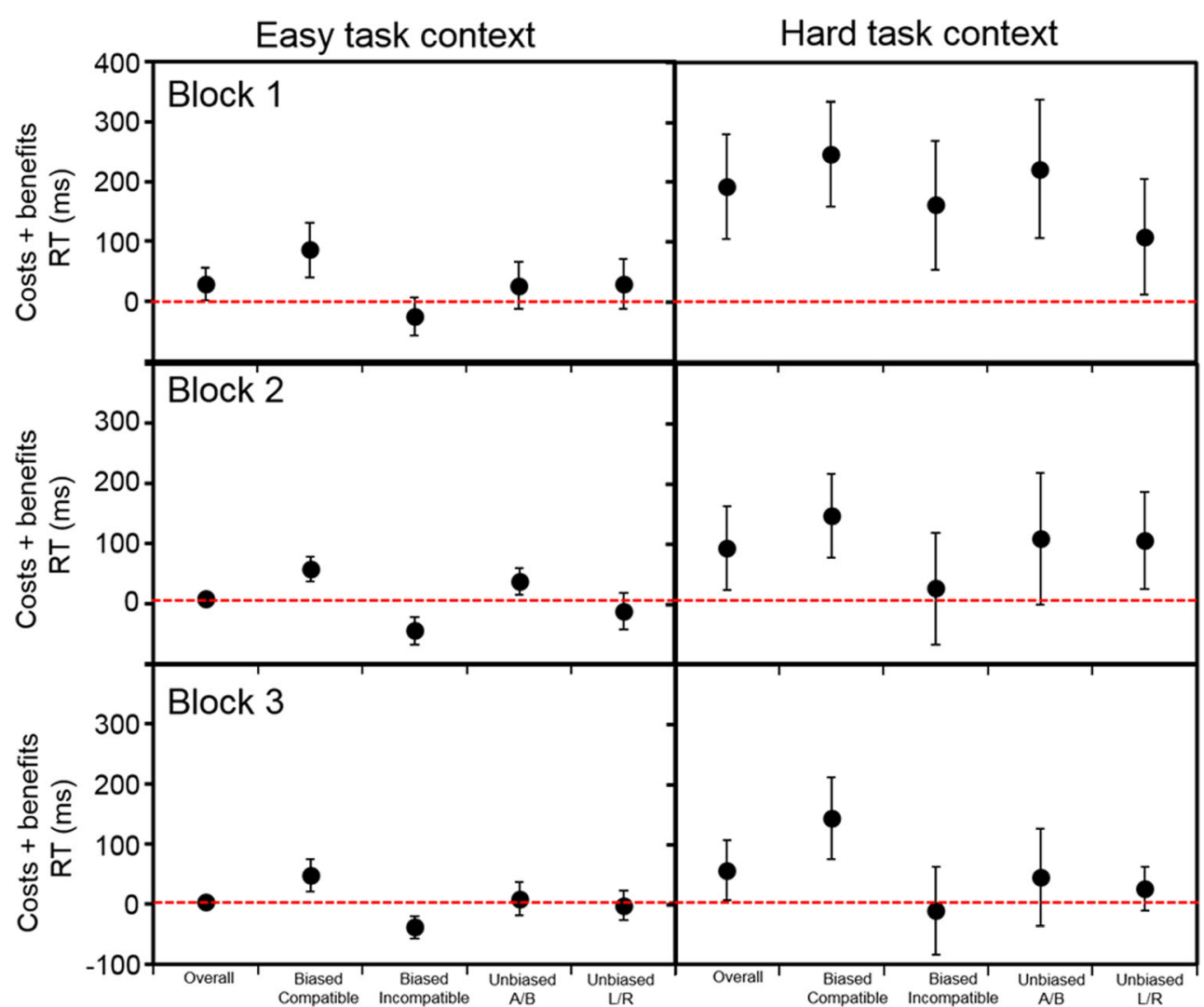

Fig. 4 Costs-plus-benefits (invalid RT-valid RT) in the overall, biased compatible, biased incompatible, unbiased above/below, and unbiased left/right categories of Experiment 3 as a function of task context and block. Error bars represent the $95 \%$ confidence intervals based on a within-subjects design

unbiased costs-plus-benefits also varied as a function of block. In particular, unbiased costs-plus-benefits were found to be relatively large and significant in Blocks 1 and 2 of the hard task context and then gradually decreased across Block 3 to the approximate magnitude observed in the easy task context. These findings suggested that the difficulty of searching without the aid of the spatial word cue decreased over time in the hard task context, which is likely due to improved performance after practice in performing the task, and observers' willingness to comprehend and use the spatial word cue to orient their attention decreased accordingly.

\section{General discussion}

The present study was conducted to strengthen and expand the empirical foundation for a novel hypothesis of the voluntary symbolic control of attention. According to this hypothesis, observers weigh the temporal costs of using symbolic cues to guide attention versus the temporal costs of searching without the aid of the cue. Prior to empirically testing this hypothesis, Experiment 1 confirmed that a cue-independent spatial bias, the left/right advantage, operates during visual search and facilitates shifts of attention along the horizontal axis. This effect
Summary As expected, the magnitude of the unbiased costsplus-benefits were found to be larger in the hard task context than in the easy task context, suggesting that observers were more likely to voluntarily use the $70 \%$-valid spatial word cues to guide their attention when the difficulty of searching without this cue increased. In addition, the magnitude of these 


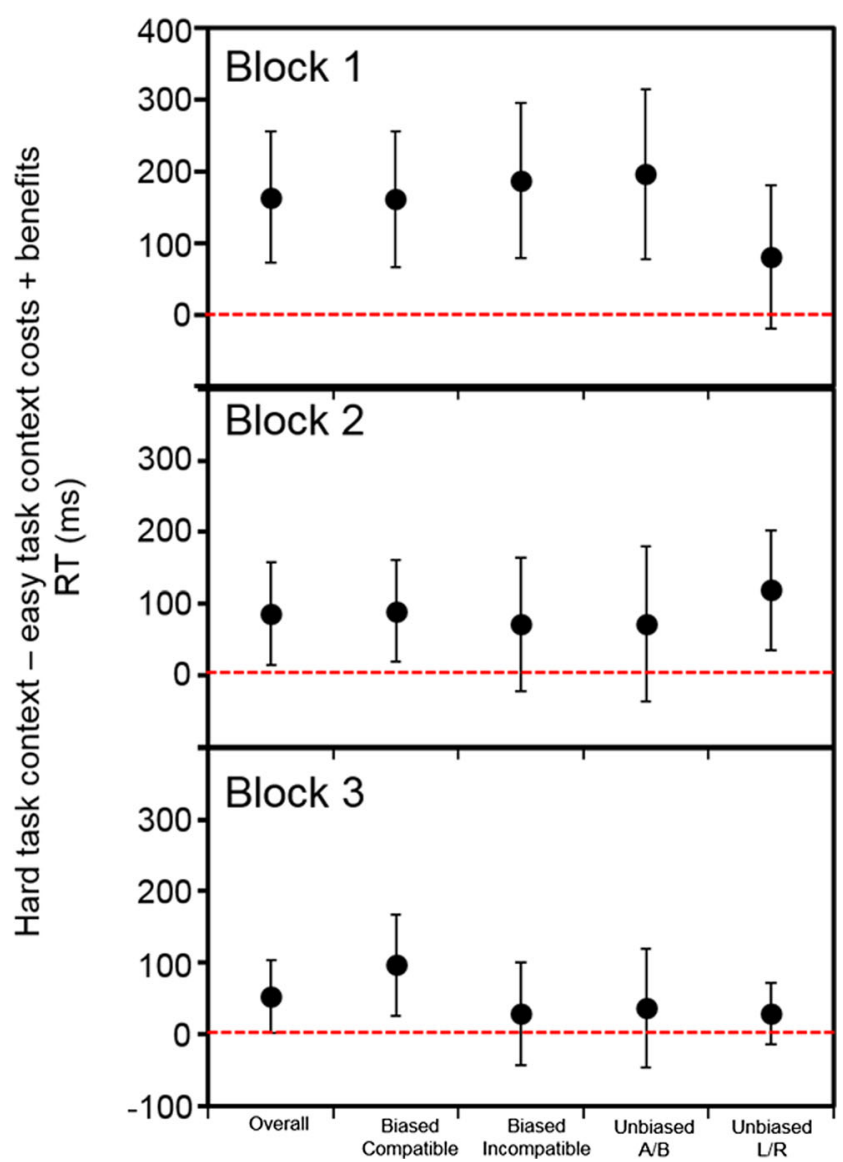

Fig. 5 The differences between the hard task context and easy task context costs-plus-benefits in the overall, biased compatible, biased incompatible, unbiased above/below, and unbiased left/right categories of Experiment 3 in each of the three block conditions. Error bars represent the $95 \%$ confidence intervals based on a between-subjects design is currently thought to be a product of the anatomical structure of the eye, which is elongated along the horizontal plane (Kobayashi \& Kohshima, 1997, 2001a, b; Nomura et al., 2005). The identification of this cue-independent bias is important because, if left uncontrolled, it can influence commonly used measures of cue processing like costs-plus-benefits and the cued axis effect (see Yantis, Meyer, \& Smith, 1991, for a detailed discussion of mixture distributions).

The influence of the cue-independent left/right advantage on these commonly used measures of cue processing was observed in Experiment 2 (see also the supplementary materials for Experiment 3). Consistent with our predictions, the overall and biased compatible costs-plus-benefits were significant and positive, suggesting that observers were using the cues to orient their attention towards the target. In contrast, the biased incompatible costs-plus-benefits were significant and negative, suggesting that invalid trials were actually faster than valid trials when the vertical axis was cued, but the target actually appeared on the horizontal axis. Fortunately, unbiased costs-plus-benefits could also be derived that neutralized the effects of the left/right advantage. These unbiased costs-plusbenefits suggested that the orientation of attention was not significantly influenced by the meaning of the cues and, therefore, that the significant overall, biased compatible, and biased incompatible costs-plus-benefits likely arose primarily due to influence of the left/right advantage on those measures.

Experiment 3 manipulated the difficulty of the search task in order to increase the extent to which observers comprehended the $70 \%$-valid spatial word cues and used them to orient their attention toward the target. Consistent with our expectations, the magnitude of unbiased costs-plus-benefits was found to increase as a function of task difficulty, despite

Table 5 Test statistics for the full 2 (vertical vs. horizontal) $\times 2$ (valid vs. invalid) $\times 3$ (Block 1 vs. Block 2 vs. Block 3$) \times 2$ (easy vs. hard) mixed ANOVA with respect to the unbiased costs-plus-benefits for Experiment 3. Mean correct response times (RTs) (ms) were used as the dependent variable

\begin{tabular}{|c|c|c|c|c|c|}
\hline Effect & $d f_{1}$ & $d f_{2}$ & $F\left(d f_{1}, d f_{2}\right)$ & $p$ & $\eta_{\mathrm{p}}^{2}$ \\
\hline Cued axis & 1 & 38 & 63.51 & $<.001$ & .63 \\
\hline Validity & 1 & 38 & 15.55 & $<001$ & .29 \\
\hline Block & 2 & 76 & 43.63 & $<.001$ & .53 \\
\hline Task context & 1 & 38 & 40.86 & $<.001$ & .52 \\
\hline Cued axis $\times$ Validity & 1 & 38 & 3.35 & .075 & .08 \\
\hline Cued axis $\times$ Block & 2 & 76 & .05 & .953 & $<.01$ \\
\hline Cued axis $\times$ Task context & 1 & 38 & 1.91 & .175 & .05 \\
\hline Validity $\times$ Block & 2 & 76 & 8.86 & $<.001$ & .19 \\
\hline Validity $\times$ Task context & 1 & 38 & 8.96 & .005 & .19 \\
\hline Block $\times$ Task Context & 2 & 76 & 14.78 & $<.001$ & .28 \\
\hline Cued axis $\times$ Validity $\times$ Block & 2 & 76 & .64 & .530 & .02 \\
\hline Cued axis $\times$ Validity $\times$ Task context & 1 & 38 & .54 & .466 & .01 \\
\hline Cued axis $\times$ Block $\times$ Task context & 2 & 76 & .60 & .553 & .02 \\
\hline Validity $\times$ Block $\times$ Task context & 2 & 76 & 4.11 & .020 & .10 \\
\hline Cued axis $\times$ Validity $\times$ Block $\times$ Task context & 2 & 76 & 2.56 & .084 & .06 \\
\hline
\end{tabular}


the spatial validity of the cue remaining constant. In addition, these findings also showed that the unbiased costs-plusbenefits were generally largest during the initial blocks and decreased thereafter, presumably because the search task gradually became easier with practice. Future research could investigate whether these block effects can be attributed to some extraneous factor, like semantic satiation (Smith \& Klein, 1990). However, the magnitude of the cued axis effect has not been shown to decrease across blocks when cue processing is mandatory (e.g., Gibson et al., 2009); thus, it is likely that this gradual decrease in evidence for cue processing across blocks reflects a change in observers' incentive to voluntarily comprehend the cues and use them to orient their attention.

The present findings have several important implications for other findings reported in the literature. First, consider the horizontal and vertical cue conditions used in Nomura et al.'s (2005) study. In both conditions, the costs-plus-benefits of shifting attention were estimated by comparing valid vertical or valid horizontal RTs to the average of both invalid opposite and invalid orthogonal RTs. This means that the magnitude of the costs-plus-benefits estimated in the horizontal cue condition consisted of both unbiased and biased compatible costs-plusbenefits; whereas, the magnitude of the costs-plus-benefits estimated in the vertical cue condition consisted of both unbiased and biased incompatible costs-plus-benefits. Accordingly, costs-plus-benefits were likely overestimated in the horizontal cue condition and underestimated in the vertical cue condition, thereby leading to their potentially false inference that uninformative horizontal cues were more potent than uninformative vertical cues. Indeed, the present findings provide no evidence that $70 \%$-valid horizontal cues were more potent than $70 \%$ valid vertical cues once unbiased costs-plus-benefits were analyzed separately from biased costs-plus-benefits.

Second, previous research showing that highly valid spatial word cues can elicit significant costs-plus-benefits (e.g., Mayer \& Kosson, 2004; Vecera \& Rizzo, 2004; see also Wright \& Ward, 2008) likely occurred not because of the spatial validity of the cues itself but rather because observers were provided with dedicated cue processing time (i.e., a non-zero SOA was inserted between the cue and the target display), which allowed them to incur the temporal cost of processing the cue before the target display appeared (Davis \& Gibson, 2012). More generally, previous research has also suggested that spatial prepositions such as above, below, left, and right are useful because these terms allow humans to communicate about the spatial layout of the external world, and that the primary purpose of such communication is to orient speech partners to relevant information in the world (Tomasello \& Call, 1997). However, many studies that have examined the relation between language and attention have used mandatory search tasks in which language comprehension is required to complete the task (see, e.g., Logan, 1995; Spivey, Tyler, Eberhard, \& Tanenhaus, 2001; see also Gibson \& Sztybel,
2014, for a review), and these studies are not relevant to voluntary attention control. Based on the evidence provided by the present study, the decision to voluntarily use such spatial prepositions to orient attention is multifaceted and highly contextual.

Third, the present findings also temper some of the findings reported by Davis and Gibson (2012). For instance, a significant left/right advantage was observed in Experiment 2 when the spatial validity of the spatial word was $70 \%$. This suggests that observers comprehended the cues less in the present study than in Davis and Gibson's study, which used 100\%-valid spatial word cues and found a nonsignificant effect of cued axis. Furthermore, the lack of significant unbiased costs-plusbenefits corroborated the conclusion that very little, if any, cue processing occurred in Experiment 2. Thus, the nonsignificant cued axis effect observed by Davis and Gibson is consistent with a reduction, but not elimination, of cue comprehension; otherwise, they should have observed a significant left/right advantage in their study as well.

Although the present study provided strong evidence that only unbiased costs-plus-benefits should be used to evaluate the status of voluntary shifts of attention, the selective use of these within-axis trials required discarding approximately twothirds of the invalid trials (i.e., the orthogonal invalid trials). One way to remedy this situation would be to remove orthogonal target locations altogether and only present stimuli on the same axis. For example, experimental blocks could be comprised of trials where targets are only presented at the valid and invalid opposite locations along the vertical or horizontal axis. This approach would experimentally eliminate the axis asymmetry caused by the left/right advantage and allow for both conditions to be compared directly. However, we expect that this approach would also influence observers' willingness to use the cues because the number of potential target locations in each block would be reduced from four to two. This reduction in the number of distractors would reduce the temporal costs of performing an unguided search for the target, thereby decreasing observers' willingness to use the cues. Future studies should investigate the most effective experimental approach toward neutralizing the left/right advantage within the spatial cuing paradigm.

Finally, consideration of an alternative hypothesis regarding the results of the present study should be addressed. According to this alternative account, observers in the present study voluntarily comprehended the spatial word cues and freely searched for the only target letter present in each display simultaneously. Thus, in contexts where the target was relatively easy to locate and discriminate without the aid of the cue (e.g., in Experiment 2 and in the easy task context of Experiment 3), the search for the target without the aid of the cue, and thus the response itself, may have been completed before any measureable effects of cue processing could be detected, even though cue processing did indeed occur. This alternative hypothesis differs from the hypothesis offered in the present study because it suggests that observers did not 
voluntarily adapt their task strategy to account for task difficulty.

Although it is difficult to address this alternative hypothesis in the present study, evidence against it was reported by Davis and Gibson (2012), who observed a cued axis effect using a 0ms cue-target SOA in their unambiguous display condition when the experimental block was composed of a random mixture of both unambiguous and ambiguous displays, but not when the experimental block was composed of only unambiguous displays (see Fig. 1). If observers were simultaneously processing the cues and freely searching for the only target letter present in each unambiguous display, then the same cued axis effect (or lack thereof) should have been observed in both contexts. However, this was not the case, which led Davis and Gibson (2012) to the conclusion that differences in voluntary cue processing observed in these contexts varied as a function of the cue processing strategy of the observer, and did not simply reflect the physical constraints imposed by the different displays.

Based on Davis and Gibson's (2012) previous findings, the results of the present study were interpreted to support the hypothesis that, in contexts where the target was relatively easy to locate and discriminate, observers voluntarily adapted their task strategy in an effort to avoid the relatively cumbersome process of comprehending and using the cues. That is, when observers perceived the temporal costs of using the cues to be more than the temporal costs of searching for the target without the aid of the cues, they voluntarily refrained from comprehending and using the cues. Hence, although the alternative hypothesis that cue comprehension can occur without cue utilization may be supported by future studies, we do not consider it to be a viable explanation of the present results.

In summary, the present study showed that a cueindependent left/right advantage operates in the spatial cuing paradigm and can influence commonly used measures of cue comprehension if left uncontrolled. Moreover, the present study challenged the monolithic assumption that high spatial validity inevitably leads to voluntary symbolic control by proposing that observers will voluntarily use symbolic cues to orient their attention in space when the temporal costs of using the cues are perceived to be less than the temporal costs of searching without the aid of the cues. The factors that might influence the temporal costs of using symbolic cues include, but are not limited to, the type of symbolic cue, the spatial validity of the cue (including potential dynamic modulation on a trial-by-trial basis; see, e.g., Arjona \& Gómez, 2013), and the duration of dedicated cue-processing time (cue-target SOA). Conversely, the factors that might influence the temporal costs of searching without the aid of the cue include, but are not limited to, task difficulty and target ambiguity. Taken together, these findings provide a new standard for evaluating spatial cuing effects and shed light on the nature of voluntary attention control.

\section{References}

Arjona, A., \& Gómez, C. M. (2013). Sequential effects in the central cue Posner paradigm: On-line Bayesian learning. In G. R. Mangun (Ed.), Cognitive electrophysiology of attention: Signals of the mind (pp. 45-57). Amsterdam: Elsevier.

Bryant, D. J., Tversky, B., \& Franklin, N. (1992). Internal and external spatial frameworks for representing described scenes. Journal of Memory and Language, 31(1), 74-98.

Carlson, L. A., \& Van Deman, S. R. (2008). Inhibition with a reference frame during the interpretation of spatial language. Cognition, $106(1), 384-407$.

Carrasco, M., Talgar, C. P., \& Cameron, E. L. (2001). Characterizing visual performance fields: Effects of transient covert attention, spatial frequency, eccentricity, task and set size. Spatial Vision, 15(1), $61-75$.

Corballis, M. C. (1988). Recognition of disoriented shapes. Psychological Review, 95(1), 115-123.

Corbett, J. E., \& Carrasco, M. (2011). Visual performance fields: Frames of reference. PLoS ONE, 6(9), e24470.

Davis, G. J., \& Gibson, B. S. (2012). Going rogue in the spatial cuing paradigm: High spatial validity is insufficient to elicit voluntary shifts of attention. Journal of Experimental Psychology: Human Perception and Performance, 38, 1192-1201.

Dickson, C. A., \& Intraub, H. (2009). Spatial asymmetries in viewing and remembering scenes: Consequences of an attentional bias? Attention, Perception, \& Psychophysics, 71(6), 1251-1262.

Folk, C. L., \& Gibson, B. S. (2001). Attraction, distraction, and action: Interdisciplinary perspectives on attentional capture. Oxford: Elsevier.

Forster, K. I., \& Forster, J. C. (2003). DMDX: A Windows display program with millisecond accuracy. Behavior Research Methods: Instruments and Computers, 35(1), 116-124.

Franklin, N., \& Tversky, B. (1990). Searching imagined environments. Journal of Experimental Psychology: General, 119(1), 63-76.

Friesen, C., \& Kingstone, A. (1998). The eyes have it! Reflexive orienting is triggered by nonpredictive gaze. Psychonomic Bulletin and Review, 5(3), 490-495.

Friesen, C., Ristic, J., \& Kingstone, A. (2004). Attentional effects of counterpredictive gaze and arrow cues. Journal of Experimental Psychology: Human Perception and Performance, 30(2), 319-329.

Gibson, B. S., \& Bryant, T. A. (2005). Variation in cue duration reveals top-down modulation of involuntary orienting to uninformative symbolic cues. Perception \& Psychophysics, 67(5), 749-758.

Gibson, B. S., \& Davis, G. J. (2011). Grounding spatial language in the motor system: Reciprocal interactions between conceptual control and spatial orienting. Visual Cognition, 19(1), 79-116.

Gibson, B. S., \& Kingstone, A. F. (2005). Visual attention and the semantics of space: Beyond central and peripheral cues. Paper presented at the 46th annual meeting of the Psychonomic Society, Toronto, CA.

Gibson, B. S., \& Kingstone, A. (2006). Visual attention and the semantics of space: Beyond central and peripheral cues. Psychological Science, 17(7), 622-627.

Gibson, B. S., \& Sztybel, P. (2014). The spatial semantics of symbolic attention control. Current Directions in Psychological Science, 23(4), 271-276.

Gibson, B. S., Folk, C. L., Theeuwes, J., \& Kingstone, A. (2008). Attentional capture. London: Psychology Press.

Gibson, B. S., Scheutz, M., \& Davis, G. J. (2009). Symbolic control of visual attention: Semantic constraints of the spatial distribution of attention. Attention, Perception, \& Psychophysics, 71(2), 363-374.

Gray, W. D., Sims, C. R., Fu, W.-T., \& Schoelles, M. J. (2006). The soft constraints hypothesis: A rational analysis approach to resource 
allocation for interactive behavior. Psychological Review, 113(3), 461-482.

Heron, W. (1957). Perception as a function of retinal locus and attention. American Journal of Psychology, 70(1), 38-48.

Ho, C., \& Spence, C. (2006). Verbal interface design: Do verbal directional cues automatically orient visual spatial attention? Computers in Human Behavior, 22(4), 733-748.

Hommel, B., Pratt, J., Colzato, L., \& Godijn, R. (2001). Symbolic control of visual attention. Psychological Science, 12(5), 360-365.

Horowitz, T. S., Wolfe, J. M., Alvarez, G. A., Cohen, M. A., \& Kuzmova, Y. I. (2009). The speed of free will. The Quarterly Journal of Experimental Psychology, 62(11), 2262-2288.

Jonides, J. (1981). Voluntary versus automatic control over the mind's eye movement. In J. B. Long \& A. D. Baddeley (Eds.), Attention and performance $I X$ (pp. 187-203). Hillsdale: Erlbaum.

Kobayashi, H., \& Kohshima, S. (1997). Unique morphology of the human eye. Nature, 387, 767-768.

Kobayashi, H., \& Kohshima, S. (2001a). Evolution of the human eye as a device for communication. In T. Matsuzawa (Ed.), Primates origins of human cognition and behavior (pp. 383-401). Tokyo: Springer-Verlag.

Kobayashi, H., \& Kohshima, S. (2001b). Unique morphology of the human eye and its adaptive meaning: Comparative studies on external morphology of the primate eye. Journal of Human Evolution, 40(5), 419-435.

Leblanc, E., \& Jolicoeur, P. (2010). How do selected arrows guide visuospatial attention? Dissociating symbolic value and spatial proximity. Journal of Experimental Psychology: Human Perception and Performance, 36(5), 1314-1320.

Logan, G. D. (1995). Linguistic and conceptual control of visual spatial attention. Cognitive Psychology, 28(2), 103-174.

Logan, G. D., \& Sadler, D. D. (1996). A computational analysis of the apprehension of spatial relations. In P. Bloom \& M. A. Peterson (Eds.), Language and space: Language, speech and communication (pp. 493-529). Cambridge: MIT Press.

Mayer, A. R., \& Kosson, D. S. (2004). The effects of auditory and visual linguistic distractors on target localization. Neuropsychology, 18(2), $248-257$.

Nomura, M., Katahata, M., \& Hashiya, K. (2005). Visual orienting occurs asymmetrically in horizontal vs. vertical planes. Psychologia, 48(3), 205-217.

Ossandon, J. P., Onat, S., \& Konig, P. (2014). Spatial biases in viewing behavior. Journal of Vision, 14(2), 1-26.

Posner, M. I. (1980). Orienting of attention. Quarterly Journal of Experimental Psychology, 32(1), 3-25.

Posner, M. I., Snyder, C. R., \& Davidson, B. J. (1980). Attention and the detection of signals. Journal of Experimental Psychology: General, 109(2), 160-174.

Pratt, J., Radulescu, P., Guo, R. M., \& Hommel, B. (2010). Visuospatial attention is guided by both the symbolic value and the spatial proximity of selected arrows. Journal of Experimental Psychology: Human Perception and Performance, 36(5), 1321-1324.
Rinaldi, L., Di Luca, S., Henik, A., \& Girelli, L. (2014). Reading direction shifts visuospatial attention: An interactive account of attentional biases. Acta Psychologica, 151, 98-105.

Ristic, J., \& Kingstone, A. (2006). Attention to arrows: Pointing to a new direction. Quarterly Journal of Experimental Psychology, 59(11), 1921-1930.

Ristic, J., \& Kingstone, A. (2009). Rethinking attentional development: Reflexive and volitional orienting in children and adults. Developmental Science, 12(2), 289-296.

Ristic, J., Friesen, C., \& Kingstone, A. (2002). Are the eyes special? It depends on how you look at it. Psychonomic Bulletin \& Review, 9(3), 507-513.

Robertson, L. C. (2004). Space, objects, minds, and brains. New York: Psychology Press.

Smith, L., \& Klein, R. (1990). Evidence for semantic satiation: Repeating a category slows subsequent semantic processing. Journal of Experimental Psychology: Learning, Memory, and Cognition, 16(5), 852-861.

Spalek, T. M., \& Hammad, S. (2005). The left-to-right bias in inhibition of return is due to the direction of reading. Psychological Science, 16(1), 15-18.

Spivey, M. J., Tyler, M. J., Eberhard, K. M., \& Tanenhaus, M. K. (2001). Linguistically mediated visual search. Psychological Science, 12(4), 282-286

Takio, F., Koivisto, M., \& Hämäläinen, H. (2014). The influence of executive functions on spatial biases varies during the lifespan. Developmental Cognitive Neuroscience, 10, 170180.

Tipples, J. (2002). Eye gaze is not unique: Automatic orienting in response to uninformative arrows. Psychonomic Bulletin \& Review, 9. 314-318.

Tipples, J. (2008). Orienting to counterpredictive gaze and arrow cues. Perception \& Psychophysics, 70(1), 77-87.

Tomasello, M., \& Call, J. (1997). Primate cognition. New York: Oxford University Press.

Vecera, S. P., \& Rizzo, M. (2004). What are you looking at? Impaired 'social attention' following frontal-lobe damage. Neuropsychologia, 42(12), 1657-1665.

Wolfe, J. M., Alvarez, G. A., \& Horowitz, T. S. (2000). Attention is fast but volition is slow. Nature, 406(6797), 691.

Wright, R. D., \& Ward, L. M. (2008). Orienting of attention. New York: Oxford University Press.

Yantis, S. (1996). Attentional capture in vision. In A. Kramer, M. Coles, \& G. Logan (Eds.), Converging operations in the study of selective visual attention (pp. 45-76). Washington, DC: American Psychological Association.

Yantis, S., Meyer, D. E., \& Smith, J. E. K. (1991). Analyses of multinomial mixture distributions: New tests for stochastic models of cognition and action. Psychological Bulletin, 110(2), $350-374$. 